\title{
Modification of the surface plasmon enhanced optical forces on metal nanorod pairs by axial rotation and by dielectric intralayer
}

\author{
Aybike Ural Yalçın, Özgür E. Müstecaplıŏlu and Kaan Güven \\ Department of Physics, Koç University, Sarlyer, Istanbul, Turkey, 34450
}

\begin{abstract}
We investigate numerically the effect of axial rotation and the presence of a dielectric intralayer on the spectral behavior of the optical force on a gold nanorod pair. The frequency spectrum of the optical force is obtained through the Maxwell stress tensor formulation and the full vectorial solution of electromagnetic waves. The common and the relative forces, which are defined through the optical force acting on each nanorod, are computed for different axial rotations and for different permittivity and thickness of the dielectric intralayer. We found that both the misaligment and the dielectric intralayer can be utilized to tailor the magnitude and direction of the relative optical force, providing a tunable attractive or repulsive response between the nanorods.
\end{abstract}

Keywords: optical force, surface plasmon, nanooptics

\section{Introduction}

The optically generated forces on micro- and nanostructures are becoming a dependable tool in a wide range of chemical, biomedical and integrated photonics applications $[1,2,3,4,5,6,7]$. The optical forces are usually classified as gradient- and scattering-type. The gradient force is induced by the gradient of the electric component of the electromagnetic field, and it forms the basic mechanism of optical tweezers [8]. The scattering force is imparted by the momentum of the incident light. Typically, the scattering force is utilized in cavity optomechanics [9].

At present, the utilization of these forces is matured well for manipulating micron-sized particles. At nanoscales (i.e. in the deep subwavelength regime), the excitation of the surfaceplasmons (SPs) on the target particle $[10,11]$ can contribute significantly to the induced optical force. For instance, for two closely placed metallic nano-spheres, a strong enhancement in the optical force can be obtained when the frequency laser is around the SP resonance [12, 13, 14, 15].

The optical force on rod-shaped metallic nanostructures has been a subject of recent studies. These involve coupled nanowire pairs[16] or head-to-tail coupled nanorod dimers in the presence of a metal slab,[17] and coupled nanorod heterodimers[18]. The frequency spectrum of the relative optical force between the nanorods is investigated as a function of the geometrical parameters of the rods and attractive and repulsive regimes are found. The optical force on coupled nanorod shaped particles is relevant from various aspects. For instance, the optical force is the basic mechanism of optical bonding/antibonding between the particles. The excitation of surface-plasmons of the nanorods provides a local trapping field for other particles. The nanorod 
itself can be regarded as a nanoantenna[19] and its radiation properties can be modified by the optical force.

Motivated by these studies, we report the manipulation of the optical forces on a metallic nanorod pair by axial rotation of the nanorods or by introducing a dielectric spacer layer. The optical forces are obtained around the surface-plasmon enhanced electric and magnetic dipole resonances. In particular, we demonstrate that the relative force acting on the nanorods can be made positive or negative through these design parameters, which can lend itself to dynamical tuning mechanisms as well. The paper is organized as follows: In section 2 we give a brief formulation of the optical force through the Maxwell Stress Tensor. Section 3 introduces the computational model of the nano-rod pair and the simulation method. Section 4-5 presents the results obtained for different rotations and for a dielectric spacer of different dielectric constant or thickness. Section 6 concludes the paper.

\section{Methods and Theory}

Evidently, the optical forces depend on the particular geometry of the metallic nanoparticle. In view of the existing literature and the experimentally available nanorod shapes, we employ a typical model adopted from a recent study[16]. This model consists of two identical pill-shaped metal nanorods of length $L$, diameter $D$ with an axial separation of $d+D$ (see Fig. 1 rightmost panel). When present, the dielectric intralayer is a square slab of edge length $a$ and thickness $w$. The coordinate system is set such that the rods have their center on the $x$ axis. The default rod axis is in the $z$ direction. The incident electromagnetic plane wave travels in the $+x$ direction and polarized along the $z$ axis. We label the rods as 2 and 1 such that the light is incident on the 2 nd rod.

We consider two separate configurations as depicted in Fig. 1. We define the aligned nanorod pair as our reference system, and obtain its optical force spectrum for comparison. The first configuration we consider involves axial rotation of the rods. Each rod is rotated around the $x$ axis while the other is kept fixed. In the second configuration, we insert a dielectric layer between the nanorod pairs. In the calculations, the permittivity and the thickness of the intralayer is varied. In all configurations, the geometrical parameters of the rods and the distance between the rod centers are kept fixed. The nanorod material is chosen as gold with frequency dependent permittivity taken from references[20]. The dielectric intralayer is assumed to be lossless and dispersionless. While the implementation of a real dielectric material is straightforward, we are primarily interested in isolating the effect of constant permittivity of different values in our study. The loss is rather negligible though a dielectric of a few tens of nanometers thick and transparent at $\mathrm{THz}$ frequencies. The incident electromagnetic $(\mathrm{em})$ radiation wavelength ranges from $600 \mathrm{~nm}$ to $800 \mathrm{~nm}$, which suitably covers the electric and magnetic dipole resonances of the structures.

The numerical simulations are performed using a commercial software (Comsol Multiphysics) which employs a finite element solver for Maxwell's equations. The 3D model system is placed at the center of a large spherical computation domain, which is enclosed by perfectly matched layers to simulate open space (reflectionless boundary conditions). We implement a wavelengthscaled adaptively refined mesh close to the metal surfaces. The perfectly matched layer has a different mesh structure, minimizing the reflection of scattered fields back into the computational domain.

Once the convergence of the electromagnetic field solutions is obtained at a given wavelength through the simulation, the optical force on each nanorod is calculated using the Maxwell stress 

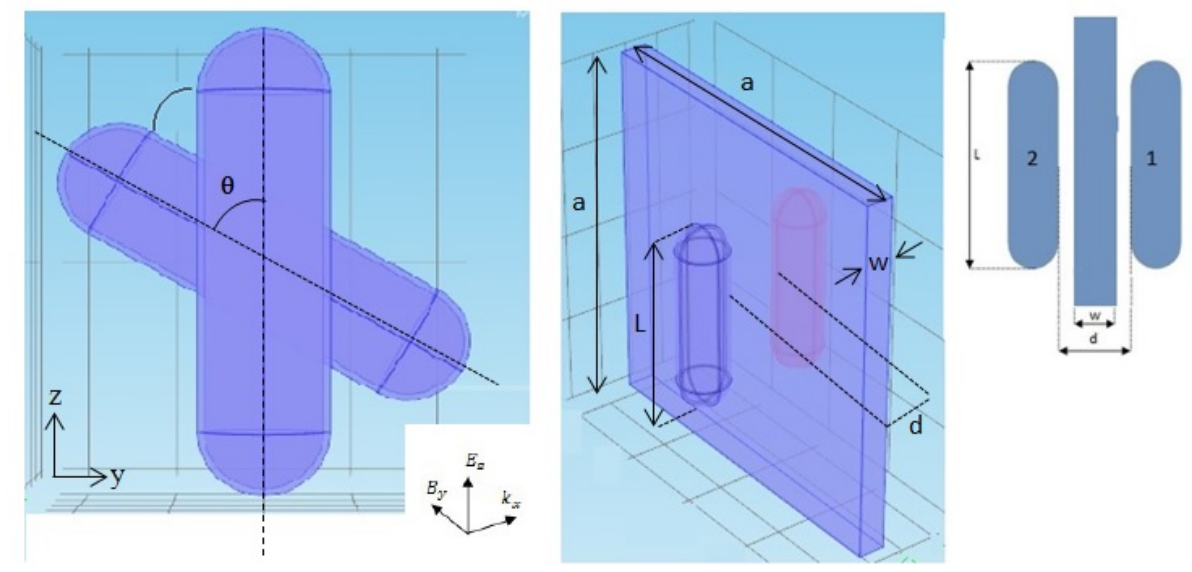

Figure 1: Schematic view of the metal nanorod pair model used in this study with two separate configurations: Axial rotation (left panel) and a dielectric intralayer (middle panel). The right panel shows the geometrical parameters of the rods and the dielectric intralayer

tensor. The $i^{\text {th }}$ component of the total Lorentz force is given by

$$
F_{i}=\iiint_{V}\left(\rho E_{i}+\epsilon_{i j k} J_{j} B_{k}\right) d V
$$

where $\epsilon_{i j k}$ is Levi-Civita symbol and $\mathrm{V}$ is a volume that encloses a nanorod. Defining force per unit volume expressed in terms of divergence of Maxwell stress tensor $\stackrel{\leftrightarrow}{T}$ and partial time derivative of Poyntings vector $\vec{S}$, the integral can be transformed to

$$
F_{i}=-\varepsilon_{0} \mu_{0} \iiint_{V} \frac{\partial S_{i}}{\partial t} d V+\iiint_{V} \frac{\partial T_{i j}}{\partial x_{j}} d V
$$

where $S_{i}=\varepsilon_{0} c^{2} \epsilon_{i j k} E_{j} B_{k}$ is Poyntings vector ( $c=$ speed of light in free space and $\varepsilon_{0}$ : vacuum permittivity) and

$$
T_{i j}=\varepsilon_{0}\left(E_{i} E_{j}-\frac{1}{2} \delta_{i j} E^{2}\right)+\frac{1}{\mu_{0}}\left(B_{i} B_{j}-\frac{1}{2} \delta_{i j} B^{2}\right)
$$

with $\delta_{i j}$ Kronecker delta.

In the steady state, the time average of the first term on the right-hand side of Eq. (2) vanishes. The second term can be converted to a flux integral

$$
\left\langle F_{i}\right\rangle=\oiint_{S}\left\langle T_{i j}\right\rangle n_{j} d S
$$


over an integration surface $S$ that encapsulates each nanorod in separate where $n_{j}$ is the outward surface normal in the $j^{\text {th }}$ direction. Henceforth, we consider only the $x$-component of the force which is the component in the direction of the incident electromagnetic wave.

The enhancement of the optical force is directly associated with the electric and magnetic dipole moments of the nanorod pair. The electric dipole moment is given by

$$
p_{z}=\iiint_{V} P_{z} d V=\iiint_{V}\left(D_{z}-\varepsilon_{0} E_{z}\right) d V
$$

where $P_{z}$ is the z-component of the polarization and $D_{z}$ is the z-component of the displacement field. The magnetic moment is calculated from the rotation of the polarization currents $r \times J_{p}$ ( $\mathrm{r}$ is the position vector pointing the volume element that contains the current density from the origin which is the center of mass (CM) of the nanorod pair ), which yields

$$
m_{y}=\iiint_{V}\left(z \frac{\partial P_{x}}{\partial t}-x \frac{\partial P_{z}}{\partial t}\right) d V=-i \omega \iiint_{V}\left[z\left(D_{x}-\varepsilon_{0} E_{x}\right)-x\left(D_{z}-\varepsilon_{0} E_{z}\right)\right] d V
$$

where $\omega$ is the frequency of the time harmonic fields.

\section{Optical force in the gold nanorod pair (reference system)}

We first calculate the reference force spectrum of the gold nanorod pair in vacuum, as in Ref[16]. The geometrical parameters are $D=25 \mathrm{~nm}, L=100 \mathrm{~nm}$ and $d=35 \mathrm{~nm}$. We define $F_{1}$ and $F_{2}$ as the respective optical force on each nanorod. The common force $F_{\text {common }}=\left(F_{1}+F_{2}\right)$ is the force acting on the center of mass. The relative force is defined as $F_{\text {relative }}=\left(F_{1}-F_{2}\right) / 2$. The sign of the relative force implies an attractive/repulsive behavior of the rods with respect to the center of mass.

Figure 2(a) shows the calculated common- and relative force spectra of the reference system. Both the common and the relative force exhibit peaks at $422 \mathrm{THz}$ and $445 \mathrm{THz}$. While the common force is all positive, the relative force takes negative and positive values respectively at the peak frequencies. The electric and magnetic dipole moments plotted in Fig. 2(b)-(c) indicate that the peaks in the force spectrum are associated with the dipole excitations of the nanorod pair. These resonances have already been investigated in the quasistatic limit (i.e. when $\lambda>>L, D$ for metallic nanostructures $[10,11]$. The antisymmetric eigenmode at $422 \mathrm{THz}$ corresponds to the magnetic dipole moment maximum and leads to an attractive relative force, since the antisymmetric mode causes a charge distribution such that the charges with opposite sign accumulates at the same end of each nanorod Fig. 3 a. In contrast, the symmetric eigenmode at $445 \mathrm{THz}$ is the electric dipole moment maximum and results in the repulsive relative force. The dependence of the optical force to the geometrical parameters of the nanorod pair, in particular to the rod length and the inter-rod distance are discussed in Ref.[16].

Figure 2(a) also shows the force when the nanorods are perfect electric conductors (PEC) (green and black solid lines). This indicates that the optical force is significantly enhanced in the presence of surface-plasmon resonances.

\section{Optical force on nanorod pair with axial rotation}

We now consider a structural modification in which one rod is rotated about the $x$ axis as shown in Fig. 1. Evidently, the hybridized resonances of the entire system will change under this 

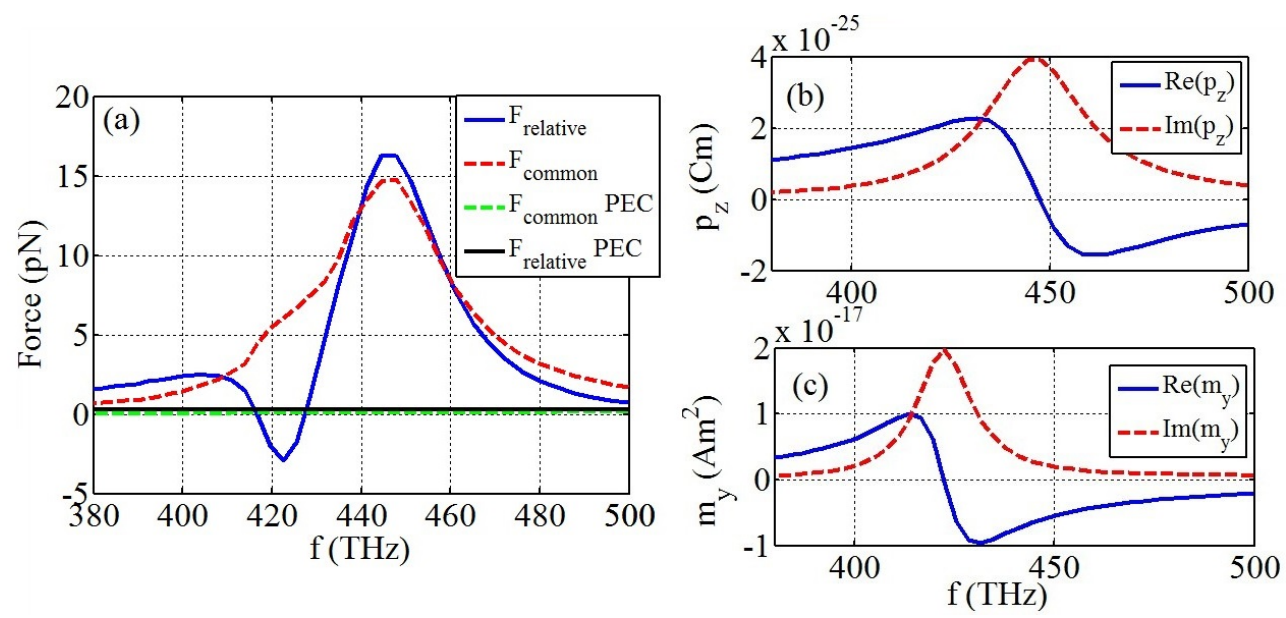

Figure 2: (a) The frequency spectra of the relative (solid blue curve) and common (dashed red curve) force in the nanorod pair system. The nanorods are of length $\mathrm{L}=100 \mathrm{~nm}$, diameter $\mathrm{D}=25 \mathrm{~nm}$ and inter-rod distance $\mathrm{d}=35 \mathrm{~nm}$. The common (black solid curve )and relative force (green dashed curve) for PEC. (b-c) The real (solid blue curve) and imaginary (dashed red curve) parts of the (b) electric and (c) magnetic dipole moment of the nanorod pair.

(a)

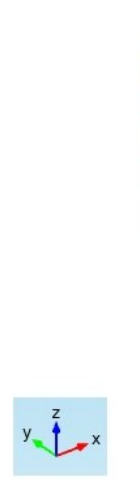

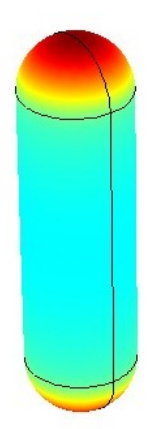

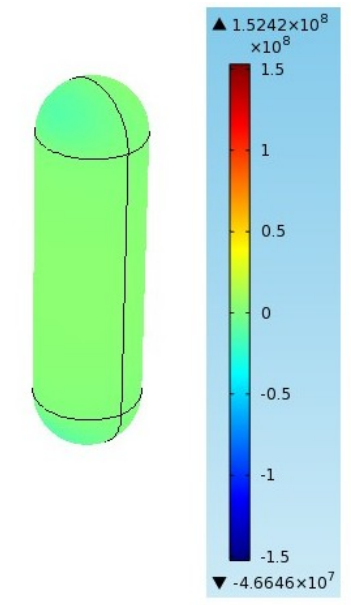

(b)

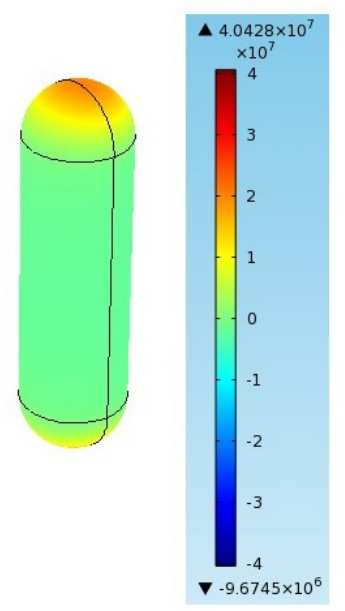

Figure 3: (a)The $E_{z}$ is plotted at magnetic dipole resonance (422 THz) corresponding to an attractive force. (b) The $E_{z}$ is plotted at the electric dipole resonance $(445 \mathrm{THz})$ resulting in a repulsive force.

rotation. Since the coupling is most prominent between the rod tips, a rotation will decrease the coupling, and hence the resonance strength. This expected behavior is confirmed by the results shown in the common force spectra (Fig. 4 (b), (d)) where the resonance peaks are red-shifted and decreased. On the other hand, both the magnitude and the direction of the relative force depend on which rod is being rotated and by what degree. In particular, the orthogonal rotation of the first rod results in a weak but all-negative relative force (Fig. 4 (a), black dotted line) whereas the rotation of the second rod yields an all positive relative force (Fig. 4 (c), black dotted line). The orthogonally rotated rod becomes "dark" and the optical force on the system is dominated by 
the force acting on the "bright"" rod. Thus the bright nanorod is pushed towards or away from the dark nanorod depending on their ordering with respect to the incident light.
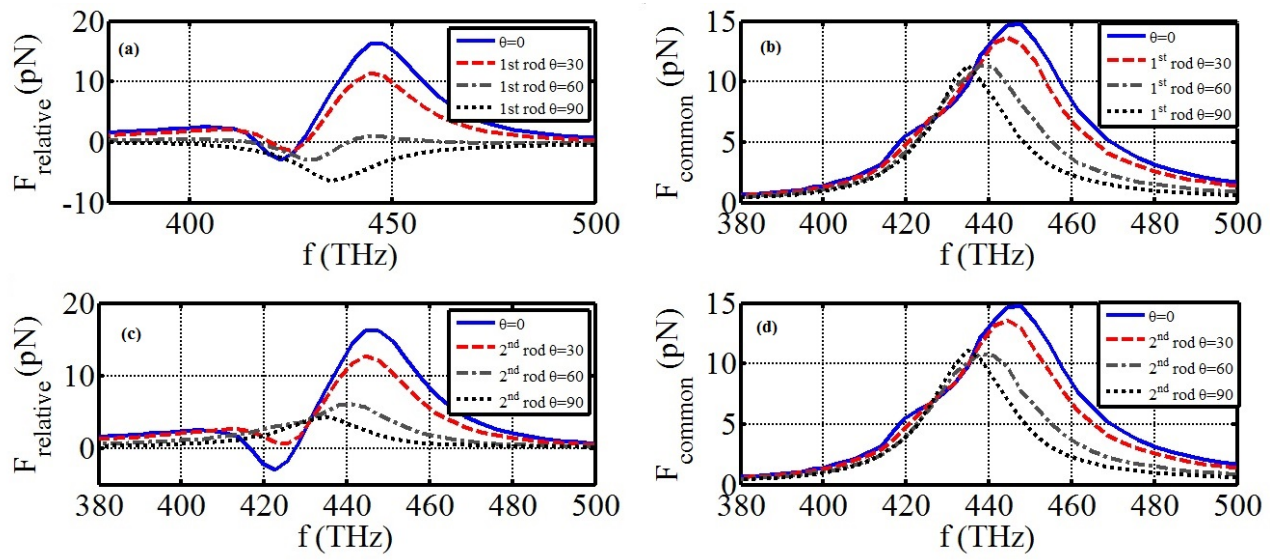

Figure 4: The (a) relative and (b) common force spectra when the $1^{s t}$ nanorod is rotated by $\theta$ around the $x$ axis. (c) and (d) show the force spectra for the rotation of the $2^{\text {nd }}$ nanorod.

In passing, we ought to note that the broken symmetry in the plane of normal incidence, the lateral forces $\left(F_{y}, F_{z}\right)$ may not vanish, leading to nonzero torque acting on the rotated nanorod. We will exclude the analysis of this torque from the present discussion.

\section{Optical force on nanorod pair in the presence of a dielectric intralayer}

A dielectric layer inserted between the nanorods will modify the local optical field distribution and hence the optical force. We consider a square shaped layer of edge length $a=200$ and characterize the optical force for the different thickness, $w$, of the layer. Figure 5 shows that increasing the thickness induces a red shift and enhancement of the force peaks that are associated with the electric and magnetic dipole resonances. While the change in the common force spectra is monotonic, the change in the sign of the relative force at the electric dipole resonance may not be deduced solely from the symmetry of the excitation mode of the nanorods as it was in the absence of the dielectric layer. The polarization of the dielectric layer contribute to the local field distribution at the nanorod surfaces. The relative force at the magnetic dipole resonance remains attractive and increases in magnitude. Since the magnetic dipole is based on an effective current loop between the nanorods, the dielectric layer is contributing by a displacement current. Increasing the thickness of the dielectric layer enlarges the current loop and hence the magnitude of the optical force.

The sign reversal of the relative optical force can also be achieved through by changing the permittivity of the dielectric layer. In fact, both the permittivity and the layer thickness serve for intensifying the electromagnetic field energy present between the nanorods. From an engineering point of view, the layer thickness is restricted by the distance between the nanorods (which should be small for an efficient coupling) whereas the permittivity is limited to the available dielectric material. The thickness of the dielectric is a fixed parameter but a tunable optical force may be achieved by incorporating a birefringent liquid crystal whose index of refraction can be tuned between two values by an external electric field. 

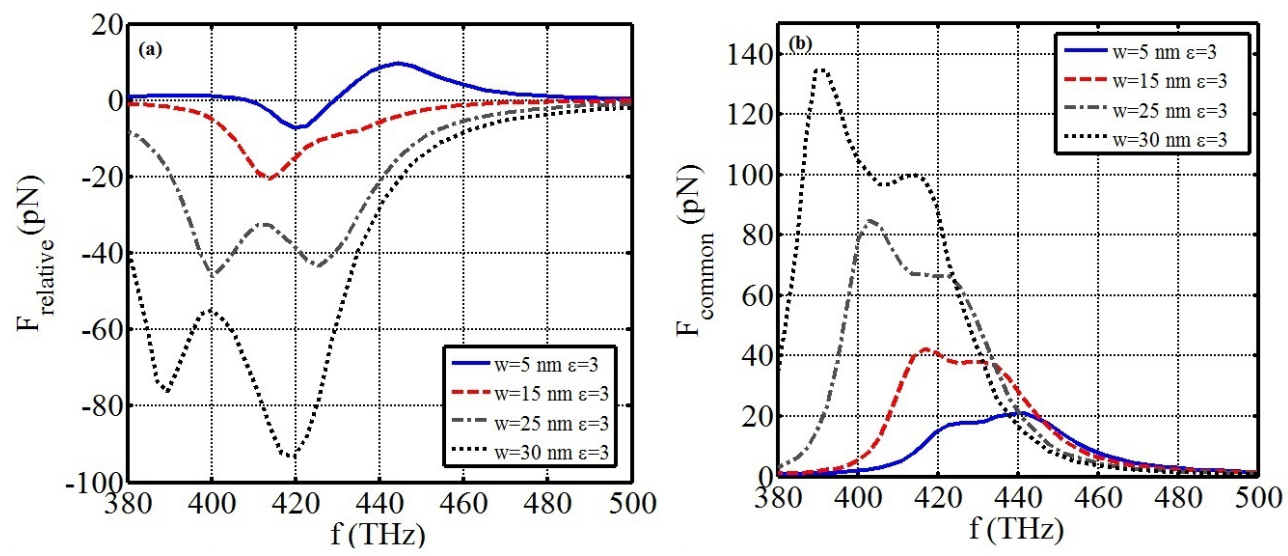

Figure 5: The (a) relative and (b) common force spectra of nanorod pair with a dielectric intralayer of constant permittivity $\varepsilon=3$ and different thicknesses $w$.

\section{Conclusion}

In this paper, we investigated numerically the modification of the surface plasmon enhanced optical forces acting on a nanorod pair by introducing rotational misalignment and by a dielectric intralayer. In both cases, the relative force can be altered to become attractive or repulsive between the nanorods. The electric and magnetic dipole resonances of the coupled nanorods provide significant the optical force, which can be further enhanced by a dielectric intralayer. Incorporating a liquid crystal layer may lead to a tunable optical force, provided that the external tuning electric field can be implemented unobtrusively to the nanostructure.

\section{Acknowledgment}

This work is supported by the Science and Technology Research Council of Turkey (TÜBITAK) under Project no. 111T285. K. Güven acknowledges partial support from the Turkish Academy of Sciences.

\section{References}

\section{References}

[1] J.-W. Tsai, B.-Y. Liao, C.-C. Huang, W.-L. Hwang, D.-W. Wang, A. E. T. Chiou, C.-H. Lin, Applications of optical tweezers and an integrated force measurement module for biomedical research, volume 4082, 2000, pp. $213-221$. URL: http://dx.doi.org/10.1117/12.390549. doi:10.1117/12.390549.

[2] D. J. Stevenson, F. Gunn-Moore, K. Dholakia, Light forces the pace: optical manipulation for biophotonics, Journal of Biomedical Optics 15 (2010) 041503-041503-21.

[3] D. Van Thourhout, J. Roels, Optomechanical device actuation through the optical gradient force, Nature Photonics 4 (2010) 211-217.

[4] A. Einat, U. Levy, Analysis of the optical force in the micro ring resonator, Optics Express 19 (2011) 20405

[5] V. Ginis, P. Tassin, C. M. Soukoulis, I. Veretennicoff, Enhancing optical gradient forces with metamaterials, Physical Review Letters 110 (2013) 057401. 
[6] L.-C. Hsu, T.-C. Chen, Y.-T. Yang, C.-Y. Huang, D.-W. Shen, Y.-T. Chen, M.-C. M. Lee, Manipulation of microparticles through optical interference patterns generated by integrated photonic devices, Lab on a Chip 13 (2013) 1151-1155.

[7] S. J. R. Staton, S. Y. Kim, S. J. Hart, G. E. Collins, A. Terray, Pico-force optical exchange (pico-FOX): utilizing optical forces applied to an orthogonal electroosmotic flow for particulate enrichment from mixed sample streams, Analytical Chemistry 85 (2013) 8647-8653.

[8] K. Svoboda, S. M. Block, Biological applications of optical forces, Annual Review of Biophysics and Biomolecular Structure 23 (1994) 247-285. PMID: 7919782.

[9] T. J. Kippenberg, K. J. Vahala, Cavity optomechanics: Back-action at the mesoscale, Science 321 (2008) $1172-$ 1176. PMID: 18755966

[10] F. Wang, Y. R. Shen, General properties of local plasmons in metal nanostructures, Physical Review Letters 97 (2006) 206806

[11] G. W. Bryant, F. J. Garcia de Abajo, J. Aizpurua, Mapping the plasmon resonances of metallic nanoantennas, Nano Letters 8 (2008) 631-636.

[12] P. Chu, D. L. Mills, Laser-induced forces in metallic nanosystems: The role of plasmon resonances, Physical Review Letters 99 (2007) 127401.

[13] K. Halterman, J. M. Elson, S. Singh, Plasmonic resonances and electromagnetic forces between coupled silver nanowires, Physical Review B 72 (2005) 075429.

[14] J. R. Arias-Gonzalez, M. Nieto-Vesperinas, Optical forces on small particles: attractive and repulsive nature and plasmon-resonance conditions, Journal of the Optical Society of America A 20 (2003) 1201-1209.

[15] A. J. Hallock, P. L. Redmond, L. E. Brus, Optical forces between metallic particles, Proceedings of the National Academy of Sciences of the United States of America 102 (2005) 1280-1284. PMID: 15647359.

[16] R. Zhao, P. Tassin, T. Koschny, C. M. Soukoulis, Optical forces in nanowire pairs and metamaterials, Optics Express 18 (2010) 25665-25676.

[17] Q. Zhang, J. J. Xiao, X. M. Zhang, Y. Yao, Optical binding force of gold nanorod dimers coupled to a metallic slab, Optics Communications 301302 (2013) 121-126.

[18] Q. Zhang, J. J. Xiao, X. M. Zhang, Y. Yao, H. Liu, Reversal of optical binding force by fano resonance in plasmonic nanorod heterodimer, Optics Express 21 (2013) 6601-6608

[19] A. Lovera, O. J. F. Martin, Plasmonic trapping with realistic dipole nanoantennas: Analysis of the detection limit, Applied Physics Letters 99 (2011) 151104.

[20] P. B. Johnson, R. W. Christy, Optical constants of the noble metals, Physical Review B 6 (1972) 4370-4379. 